\title{
A Comprehensive Analysis and Design of Addictive Educational Mobile Games
}

\author{
Shreyas Prasad \\ Department of Information Science, \\ BMS College of Engineering \\ Bangalore, India
}

\author{
Urmika Kasi \\ Department of Information Science, \\ BMS College of Engineering \\ Bangalore, India
}

\author{
Rithika Shivakumar \\ Department of Information Science, \\ BMS College of Engineering \\ Bangalore, India
}

\begin{abstract}
Mobile game addiction is a significant aspect of consideration as there has been notable growth in mobile game usage. However, game developers face numerous obstacles with respect to educational mobile games in terms of engagement, commitment, likeability and usage. This study proposes an approach to understanding the contributors to addiction in mobile games, with the intent of designing educational games that overcome the aforementioned obstacles. These contributors were extrapolated upon analysis of the most popular games as seen from an accordingly curated questionnaire. A general user flow framework was proposed to leverage the underlying mechanisms of addiction in mobile games to make educational games more effective. Furthermore, this study expands on the general guidelines for designing addictive educational mobile games.
\end{abstract}

\section{Keywords}

Mobile Game Addiction, Educational Games, Flow, Game Mechanics

\section{INTRODUCTION}

The mobile games industry has seen exponential growth in recent times, dominating the entire gaming industry with around $60 \%$ of the market share. The industry is expected to grow to 2.7 billion worldwide users by 2021 [1][2]. This burgeoning can be justified by the numerous advantages that smartphones introduce, with regards to convenience, affordability, and ease of use, etc. It also provided a new platform for existing games to exploit, and effectively do so by incorporating features specific to smartphones themselves, such as touch screens, location services, Bluetooth and so on. Its appeal has been reflected in the widely varying age group of mobile game players, from school-going children to adults. Reports show that the average time that users play mobile games rises steadily every year [3]. Consequently, it is interesting to understand the underlying mechanisms that make games addictive.

The growth of the mobile game industry has seen significant developments in the area of game design, not only from a technological perspective but also from a psychological one. Player enjoyment is a necessary criterion to motivate continual gaming, and this depends on the mechanics (interaction with the game world), interface (design elements) and gameplay (problems and challenges). These heuristics in games were found to relate closely with the elements of flow theory [4], flow being an immersive mental state of energized focus. By understanding player behaviour and documenting their reactions to certain game features, game designers have effectively induced addiction amongst players by combining their understanding of player heuristics and have modelled game features accordingly. This incorporation of psychology with advanced mobile app development is one of the primary factors that cause addiction amongst mobile users.

Though mobile game addiction is undoubtedly rife with drawbacks and disadvantages, mobile gaming itself presents various albeit inconspicuous advantages. It promotes social interaction amongst its users and induces feelings of happiness and relaxation. From a cognitive perspective, it improves concentration and problem-solving. Additionally, it also improves motor skills and dexterity.

Although several games are markedly within the educational umbrella, their popularity has been consistently lacking. This is mainly due to the inability of game designers to effectively combine the concepts of addictive games with educational objectives. It would be appropriate, therefore, to posit that it is vital as a game designer to understand what makes mobile games addictive to create a popular gaming application. Furthermore, the requirement was also necessitated for games to increase the variety of its content to be inclusive not only of hyper-casual entertainment-oriented gameplay but also educational and cognitive understanding.

This study aims to investigate the underlying mechanisms and proponents behind addiction in mobile games and propose a conceptual framework to integrate these features for subsequent application in an educational context to create an addictive educational mobile game.

This paper is organized as follows: after this introduction in Section 1, Literature Review is explained in Section 2, Research methodology is presented in Section 3, while in Section 4, the framework model is proposed. Finally, the paper is concluded in Section 5 .

\section{LITERATURE SURVEY}

\subsection{Flow and Immersion}

In the 1970s, psychologist Mihaly Csikszentmihalyi experimentally evaluated flow theory [4] and inferred that a person's skill and the difficulty of a task to interact result in different cognitive and emotional states. Described simply, flow is an optimal experience resulting in intense engagement, heightened motivation, receptiveness to information, and diminished perception of time.

When the level of skill is low and the task too hard, people become anxious. Analogously, if the task is too easy and skill too high, people become bored. When both the skill and difficulty are at an optimum, players enter into a state of hyper-focus. While flow is a fairly new construct in the field of serious games, if harnessed effectively, it could prove to be extremely useful in attaining maximum engagement from the user and hence provide a deep learning experience. 


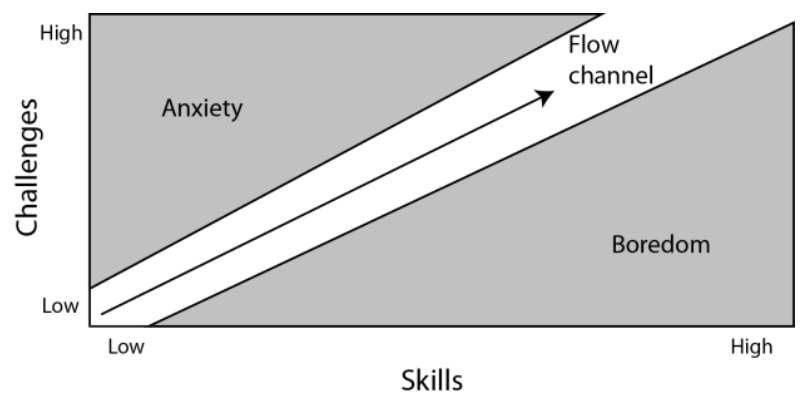

Fig 1: Flow by Mihaly Csikszentmihalyi

When looking at the features of flow, there seem to be strong links with immersion, another notion that comes along with gameplay. Seah and Cairns [5] found that the experience of flow and in-game immersion was associated with addiction.

Several games incorporate flow-like features that could act as a precursor to an increased likelihood of becoming addicted [6]. In study [7] authors have concluded that game players who have experienced flow are more likely to fall into addiction. In another study, that authors state that experience of flow has been acknowledged as an important predictor of game addictions which include mobile game addiction. They further conclude that people are prone to access media through physiological arousals, such as excitement, pleasure, and stimulation, resulting in psychological reliance on media [8].

\subsection{Mobile Game Mechanics}

Abstraction of money: The game consists of virtual currency specific to the particular game such as gold, gems, points, credits or fragments [9]. Such currency is only meaningful within the context of the game, and can be obtained by progressing through the game and finishing challenges or levels, or by exchanging real money to purchase larger quantities of such currency at a time. Authors of [9] have reviewed the status of currencies in video games and have distinguished virtual currencies in terms of game mechanics defining three types of virtual currencies. This study throws light on how VCs are used as a medium of exchange in purchases, rewards for accomplishments and as a "status indicator".

Eternal monotony: Games having eternal monotony foster long term commitment among the players. Providing large endless worlds encourages players to spend more time playing the game, which, in turn, leads to deeper involvement [10]. This is opposed to games involving a finite story arc, indicating that there is a definite end to the game, after which players have no substantial reason to play. To maintain player interest over a long term, the game must incentivize perfectionism on the player's behalf. This can be done, for example, by allowing players to repeat levels that have been completed previously without negative consequences to earn a "perfect stamp".

Authors of [11] have stated that by maintaining a comfortable pace and presenting the player with a large number of clear, intermediate goals of varying levels of difficulty that are tied to the main underlying story, longer playtime can be achieved with sustained interest. Further, authors of [12] have studied eternal monotony and concluded that this can be an immensely rewarding mechanism and psychologically engrossing one.

Reward System: These are bonuses awarded to the player for opening the game daily, with longer consecutive logins yielding higher rewards. The awarded bonuses may be in the form of the virtual currency used by the specific game. This encourages a player to open the game at least once a day to collect their rewards, which could prompt subsequent play after doing so. Daily challenges are a step further above login rewards, where players are awarded bonuses every day only if they complete a challenge. This induces longer and guaranteed playtimes, and is potentially more effective to prompt further play, since completion of the challenge results in a player's concentration and interest is piqued. Authors of [13] have given an overview of how various video game reward systems provide positive experiences to players. Further, they also say that reward systems and mechanisms in modern digital games provide social meaning for players primarily through motivation, enhanced status within gaming societies, and the use of rewards as social tools.

Artificial scarcity: This concept models items in a manner that induces difficulty in obtaining them, or makes them available only for a limited period [14]. The notion of exclusivity and scarcity increases the demand for these items and makes them more valuable in a player's eyes. This can be done by introducing "limited edition" items that may or may not be related to a current time frame such as Christmas or Halloween. Artificial scarcity is defined as the scarcity of items that exist even though either the technology for production or the sharing capacity exists to create a theoretically limitless or at least greater quantity of production than currently exists [15]. Further, authors of [16] went on to say that artificial scarcity is achieved when certain items are made difficult to obtain through gameplay when these items are most commonly not purchasable.

Multiplayer mode: Authors of [8] state that a multiplayer mode reinforces the concept of "perceived visibility", which implies that games with social aspects via online chatting or face-to-face communication contribute to flow. A multiplayer functionality enables players to view other players' game behaviour and invite friends, thereby boosting social presence and approval. Authors also state that from the perspective of social value, mobile games with social values tend to lead users to have flow experience and mobile games with social networking functions today intensify social value greatly.

Leaderboard: This is an additional, specific facet under the previously mentioned notion of "perceived visibility" [8]. A study [17] examines how leaderboard ranking affected satisfaction with a game and desire to play a game again. A leaderboard enforces social presence in the context of scores and achievements, where players may view their scores alongside other players and friends and view their standings on various platforms other than that of the game itself, such as Facebook. It can induce compulsive game playing amongst players who are highly aware of other players' expectations, in the aim of breaking records to see their names at the top.

Reminders: Sending push notifications and reminders to players enhances game visibility to draw in users. They can be used to send updates and consequently drive user retention. To make them effective, they can be structured in a format that is personalised to the player's current game status and addressing the player by their username to appropriately create a sense of urgency without annoying or spamming the user. Artificial scarcity can also be incorporated, by sending out reminders to indicate that an item is about to expire. Authors of [18] stated that the inclusion of reminders instigates players to revisit and helps overcome the forgetting curve. 
Character customization: Being able to create and customize avatars in a game creates a more personal connection between the player and the game. It also allows players to feel a larger sense of control over the game proceedings. Authors of [19] have shown that the ability to select or customize characters in educational applications and games influence factors related to learning effects such as transfer, self-efficacy, and motivation. This emphasizes that character customization has a positive effect on learning. From studies conducted by the authors of [20], identification was proven to be a strong contributor to players' positive or negative game experiences. Avatar-based customization plays an important role in players' identification with their characters by increasing their sense of autonomy.

\subsection{Educational Games}

Over recent years, the demand for educational games has increased tremendously, since they are viewed as a potentially effective tool for teaching. However, such games have not earned corresponding popularity due to an inability to incorporate key design elements to effectively create an educational game. Multiple approaches have been taken in this particular domain before.

Authors of [21] have proposed a 5-phased methodology to develop educational games centric on an underlying story while assimilating multiple different genres, involving startup, design, production, test, and post-production. An alternative approach proposes a learning objective focused method, which ties learning challenges and in-game player objectives together [22]. It involves a tight coupling with the educational curriculum and can be used for a variety of knowledge areas, provided there is at least one learning objective with which in-game objectives can be associated.

Authors of [23] investigate techniques to improve upon the effectiveness of educational games by proposing the use of fuzzy logic to make games adaptable to particular levels, making education more compliant with each individual player's capacities and learning paces. Authors of [24] consider educational design from a lower level of abstraction, and models the structure of the video game or metamodel and analyses the various relationships that exist between the different video game elements and their cardinality using UML graphical notations.

\section{RESEARCH METHODOLOGY}

\subsection{Data Collection}

This research commenced by creating a survey containing questions requiring users to stipulate their preferred games with additional questions pertaining to usage such as the duration spent playing these games daily, and playing patterns such as the frequency with which they open the apps in a day. The top 16 games were chosen for analysis. Questions were also included to understand the rationale motivating the users' playing behaviour, which was used to infer the aspects of these games that made them addictive. A total of 855 gamers completed the survey, and the results were processed and tabulated to prepare it for further analysis.

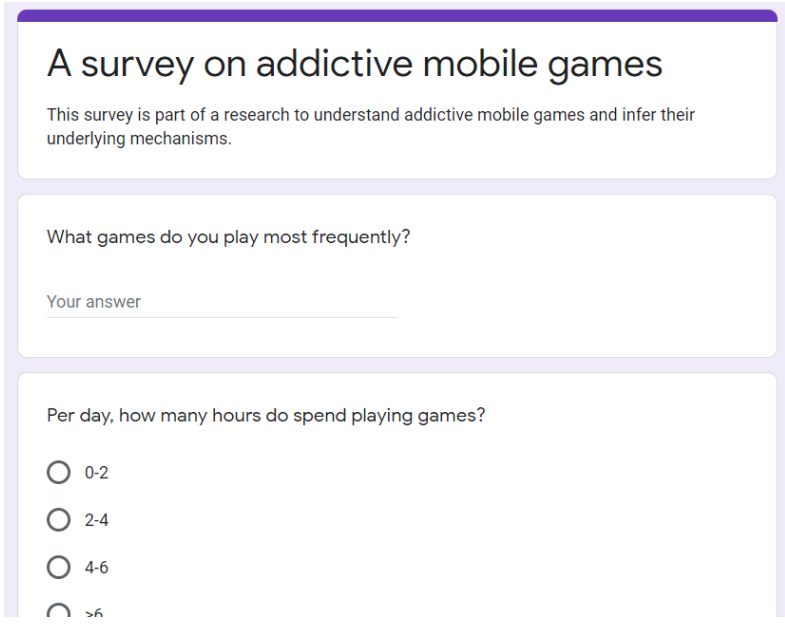

Fig 2: Snapshot of the questionnaire

\subsection{Discussion and Implications}

This study revealed that all of the chosen games used virtual currencies of some form and had multiple types of currencies within the same game. For example, in Candy Crush, gems can be used for a variety of functionalities such as replenishing lives instantly or avoid having to wait for a stipulated time. PUBG uses coins and silver fragments for making purchases within the game, where a "Royale Pass" can be bought with real money to unlock VIP benefits.

Nearly every game showed characteristics of eternal monotony, and those that didn't either consisted of an end objective that would take an extended period to complete anyway, such as Pokemon GO, or were modelled on concepts for which eternal monotony would not be applicable, such as 8 Ball Pool. However, prolonged player times can be ensured by incentivising perfectionism.

Less than half of the selected games provide a daily login reward functionality, as opposed to daily challenges, which are provided by most of the selected games. This further reinforces the presented proposition that daily challenges are potentially more effective and desirable from a game creator's perspective. In Subway Surfers, the player must collect letters of a word for every consecutive day until the word is complete to receive an additional "mystery box" upon completion, in addition to coins received daily for collecting the letter. Temple Run also consists of daily objectives, which when completed for 5 days consecutively yield "Daily Doses", and if done for 12 consecutive days yield "Daily Dozens", which are additional special rewards along with the daily coin bonus which can be used to unlock special character upgrades.

Artificial scarcity is seen in a significantly large portion of the selected games. Clash of Clans, a strategy-based game uses a combination of multiple virtual currencies (elixir, gold and gems) and artificial scarcity by making gems difficult to obtain and providing apparent advantages over gold and elixir. Clash Royale also works on a similar mechanism. Call of Duty and Fortnite present a host of limited edition accessories and items such as weapon skins and player outfits, which disappear from the in-app store after a certain period.

Most of the games also support multiplayer modes and a leaderboard feature. Zynga Poker and Ludo King stem from the necessity of multiple players. Since competitiveness and social interaction is a major driving factor for a sizable portion of players to play a game, game creators have incorporated 
these functionalities. Leaderboards are seen in most of the mentioned games, including Hill Climb Racing 2 and Fruit Ninja.

The facility to modify the player's character in a game is also present in most games, irrespective of whether an actual human avatar is involved. In Helix Jump, customizations in the form of different balls can be done whereas in games such as Jetpack Joyride, PUBG and so on, traditional character customization in the form of wardrobe and accessory makeovers can be done.

Table 1. Addictive game mechanics

\begin{tabular}{|c|c|c|c|c|c|c|c|c|c|}
\hline \multirow[b]{2}{*}{ Games } & \multicolumn{9}{|c|}{ Features } \\
\hline & 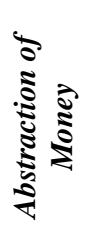 & 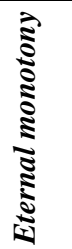 & 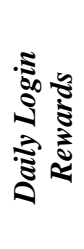 & 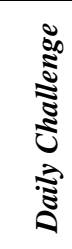 & 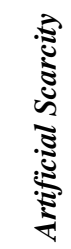 & 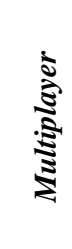 & 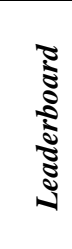 & 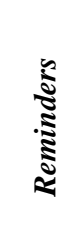 & 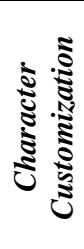 \\
\hline PubG & $\mathrm{X}$ & $\mathrm{X}$ & $\mathrm{X}$ & $\mathrm{X}$ & $\mathrm{X}$ & $\mathrm{X}$ & $\mathrm{X}$ & $\mathrm{X}$ & $\mathrm{X}$ \\
\hline Call of Duty & $\mathrm{X}$ & $\mathrm{X}$ & $\mathrm{X}$ & $\mathrm{X}$ & $\mathrm{X}$ & $\mathrm{X}$ & $\mathrm{X}$ & $\mathrm{X}$ & $\mathrm{X}$ \\
\hline Clash of Clans & $\mathrm{X}$ & $\mathrm{X}$ & $\mathrm{X}$ & & $\mathrm{X}$ & $\mathrm{X}$ & $\mathrm{X}$ & $\mathrm{X}$ & $\mathrm{X}$ \\
\hline Fortnite & $\mathrm{X}$ & $\mathrm{X}$ & & $\mathrm{X}$ & $\mathrm{X}$ & $\mathrm{X}$ & $\mathrm{X}$ & $\mathrm{X}$ & $\mathrm{X}$ \\
\hline Subway Surfers & $\mathrm{X}$ & $\mathrm{X}$ & & $\mathrm{X}$ & $\mathrm{X}$ & & $\mathrm{X}$ & $\mathrm{X}$ & $\mathrm{X}$ \\
\hline Helix Jump & $\mathrm{X}$ & $\mathrm{X}$ & & & $\mathrm{X}$ & & & & $\mathrm{X}$ \\
\hline Pokemon GO & $\mathrm{X}$ & & & $\mathrm{X}$ & & $\mathrm{X}$ & $\mathrm{X}$ & $\mathrm{X}$ & $\mathrm{X}$ \\
\hline Candy Crush & $\mathrm{X}$ & $\mathrm{X}$ & & $\mathrm{X}$ & & & & $\mathrm{X}$ & \\
\hline 8 Ball Pool & $\mathrm{X}$ & & & $X$ & & $\mathrm{X}$ & $\mathrm{X}$ & $\mathrm{X}$ & \\
\hline Fruit Ninja & $\mathrm{X}$ & $\mathrm{X}$ & & & $\mathrm{X}$ & $\mathrm{X}$ & $\mathrm{X}$ & & $\mathrm{X}$ \\
\hline Clash Royale & $\mathrm{X}$ & $\mathrm{X}$ & $\mathrm{X}$ & & $\mathrm{X}$ & $\mathrm{X}$ & $X$ & & \\
\hline Hill Climb Racing 2 & $\mathrm{X}$ & $\mathrm{X}$ & $\mathrm{X}$ & $\mathrm{X}$ & & $\mathrm{X}$ & $\mathrm{X}$ & $\mathrm{X}$ & $\mathrm{X}$ \\
\hline Temple Run & $\mathrm{X}$ & $\mathrm{X}$ & & $\mathrm{X}$ & $\mathrm{X}$ & & $\mathrm{X}$ & $\mathrm{X}$ & \\
\hline Zynga Poker & $\mathrm{X}$ & $X$ & & & & $\mathrm{X}$ & $\mathrm{X}$ & $X$ & \\
\hline Jetpack Joyride & $X$ & $\mathrm{X}$ & & $\mathrm{X}$ & & & & $\mathrm{X}$ & \\
\hline Ludo King & $\mathrm{X}$ & $\mathrm{X}$ & $\mathrm{X}$ & & $\mathrm{X}$ & $\mathrm{X}$ & $\mathrm{X}$ & & $\mathrm{X}$ \\
\hline
\end{tabular}

\section{MODEL PROPOSAL}

\subsection{Framework for User Flow}

Fig II. presents the flow of proceedings for a user of an addictive mobile game. It incorporates various mechanisms which contribute to addictive games as procured by the research conducted in the previous section.

A user is required to register themselves with the game, and must login to continue. Upon opening the app, a checking condition is fired to determine whether the user has already logged in. If they have, they are presented with the home page. If they are first time users, they are directed to a registration portal. The home page offers the user at least four main facets of use:

Leaderboard: Users can view their rankings and scores in comparison to other players locally and globally. An additional feature can display their standings with respect to their particular social circle if they logged in via a social platform, such as Google, Facebook, etc.

Profile: Users can view details pertaining to their in-game characters, such as username and current level. These details can accordingly be altered when a user desires to do so. An inventory stores the items purchased by a user from the store or won by in-game accomplishments, and these items may be enabled as per the users' requirement.

Gameplay: Users play the game in levels, progressing to the next level only if a certain threshold or criterion has been reached. Multiple levels of abstraction in terms of levels may be implemented, such as levels being included within phases, phases being included in seasons, and so on to prolong the continuity of the game in a non-linear fashion. For ease of representation and understanding, only levels have been indicated in the flow diagram. Once a level has been attempted by a user, they may progress to the next level if they successfully meet the goals set by the level, or may repeat the level to fulfil all the necessary goals. Perfectionism for eternal monotony is incentivized by allowing players to repeat games even if they have successfully met the goals for a level, but not in the best possible manner.

Store: Users can make in-game purchases using the gamespecific virtual currency. The items that can be bought may include virtual currency itself, or accessories, upgrades, outfits, or even an unlock into a different world. On entering the store, the user views the catalogue of items the game has to offer. The purchase is made using virtual currency, which, 
if not adequate, is replenished by a transaction with real money. The user may also visit the store to recharge virtual currency.

The user may choose to exit from any of these areas and return to the home page, from where they may either visit another sector or close the game.

\subsection{Implementation in Educational Games}

This study inspected prime mechanisms contributing to addiction in mobile gaming and presented a framework to incorporate these features into, thereby providing an effective setup to create an addictive educational mobile game. Gameplay should introduce a story arc in order to effectively draw in its users without being overly direct and instructive in nature. Based on the desired generality of topics to be covered by the game, it should be divided accordingly into different phases or "worlds", such that each world comprehensively covers a particular topic. These worlds should contain an appropriate number of levels, such that each level meets one or more learning objectives. Learning objectives must be clear and evaluable to effectively incorporate them into levels. To ensure a correct understanding of concepts and reinforce them, the functionality to replay levels should be given. It can also be used to enforce methodology biases, i.e., a preference for a certain procedure over others, by providing a perfect score in cases where applicable. This design, therefore, constructively includes eternal monotony.

A leaderboard and multiplayer mode should be included to introduce a social aspect and incentivize players to play further and learn more, by introducing healthy competition and social interaction as it provides a sense of accomplishment among peers. The presence of a store is mainly to boost addiction, without actually representing any learning objectives on its own. As players progress through levels and phases, they are rewarded accordingly with virtual currency, which can be used to purchase items from the store. By customizing their avatars, a personal connection is established, providing a further rationale to play the game. Furthermore, a player may also be motivated to play in the hope of acquiring a coveted item in the store. The store thus contributes to increased time of gameplay, which in turn causes learning. It also integrates the concepts of a reward system, artificial scarcity and character customisation.

\section{CONCLUSION}

This study was focused on understanding the principal hallmarks of addiction in mobile games and presenting a scheme to assimilate them accordingly into game design to create an addictive educational game. A model flow diagram was then proposed to provide a basis for the integration of the mechanisms contributing to addiction, and its application in an educational context was presented in the form of guidelines and suggestions. This model attempted to alleviate the earlier presented problems of educational games, from a conceptual perspective. Future work in the area involves the application of the proposed model and guidelines in terms of real-time development and design. A larger, more comprehensive research could be conducted to deduce more contributing factors to addiction and to gain a deeper insight into more problems of educational games and consequent potential solutions.

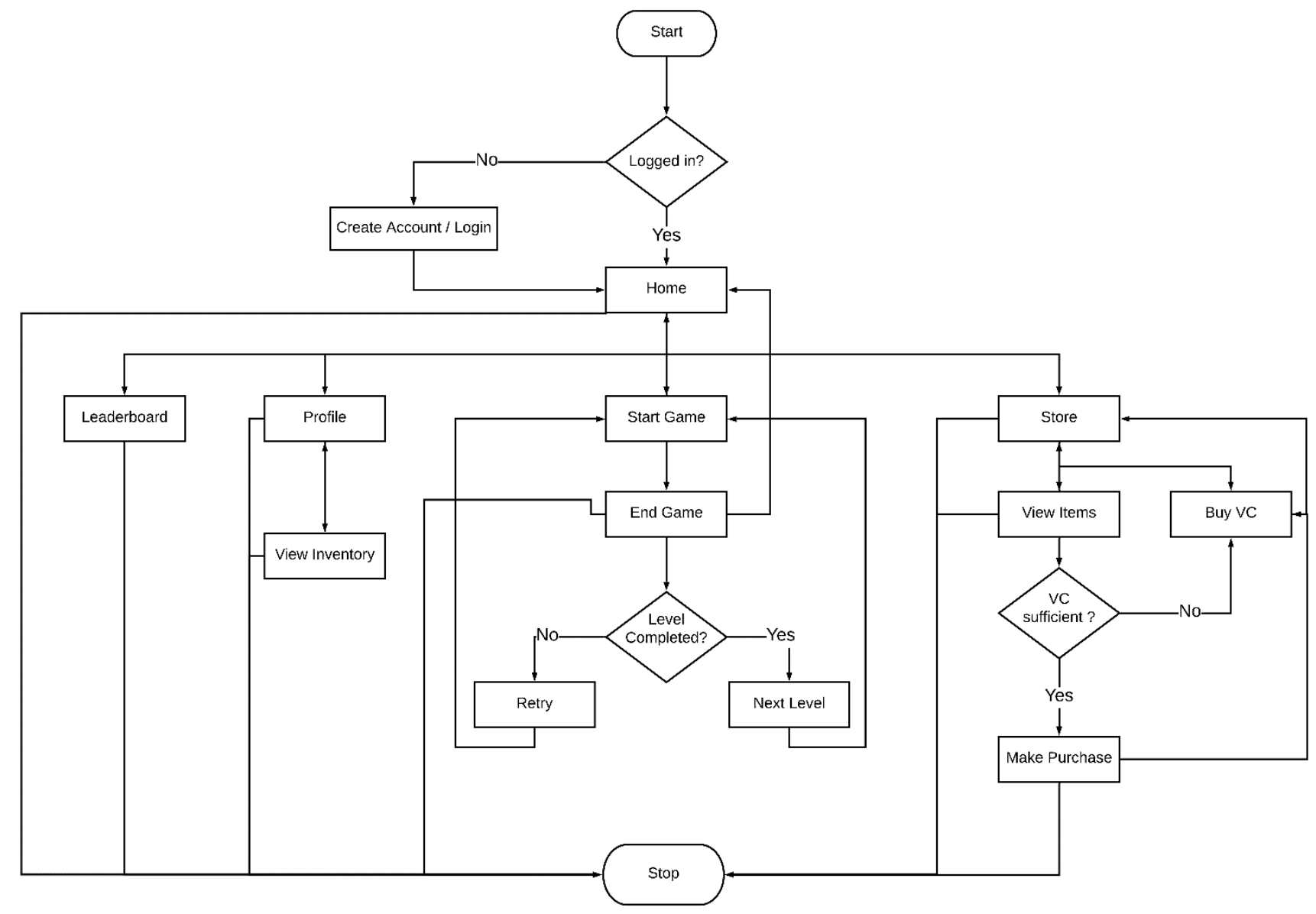

Fig 3: User flow model 


\section{REFERENCES}

[1] Gough, C. (2018, February 20). Number of mobile gamers worldwide 2014-2021, Statista, Retrieved from https://www.statista.com/statistics/748089/numbermobile-gamers-world-platform/

[2] Green, A. (2019, December 30). Mobile Gaming Generated $60 \%$ of the Global Video Games Revenue in 2019, Golden Casino News, Retrieved from https://goldencasinonews.com/blog/2019/12/30/mobilegaming-generated-60-of-the-global-video-gamesrevenue-in-2019/

[3] RealityMine , MYTH BUSTING: MOBILE GAMING DEMOGRAPHICS, MMA, Retrieved from https://www.mmaglobal.com/research/myth-bustingmobile-gaming-

demographics\#: :text=With $\% 20 \mathrm{a} \% 20$ mobile\%20device $\% 20$ always,so $\% 20$ are $\% 20$ mobile\%20gaming\%20demog raphics/

[4] Csikszentmihalyi, M., \& Csikszentmihalyi, I. S. (Eds.). (1992). Optimal experience: Psychological studies of flow in consciousness. Cambridge university press.

[5] Seah, M. L., \& Cairns, P. (2008). From immersion to addiction in videogames. People and Computers XXII Culture, Creativity, Interaction 22, 55-63.

[6] Hafeez, Maria \& Idrees, Dawood \& Kim, Jung-Yong. (2017). Game Addiction: A Brief Review. International Journal of Scientific Engineering and Research (IJSER). 5. 99-104.

[7] Chou, T. J., and Ting, C. C. (2003). The role of flow experience in cyber-game addiction. CyberPsychology and Behavior, 6(6), 663-675

[8] Sun, Y., Zhao, Y., Jia, S. Q., \& Zheng, D. Y. (2015). Understanding the Antecedents of Mobile Game Addiction: The Roles of Perceived Visibility, Perceived Enjoyment and Flow. In PACIS (p. 141).

[9] Asadi, A. R., \& Hemadi, R. (2018, November). Understanding Virtual Currencies in Video Games: A Review. In 2018 2nd National and 1st International Digital Games Research Conference: Trends, Technologies, and Applications (DGRC) (pp. 109-117). IEEE.

[10] Hoffmann, G., Martin, R., \& Weinhardt, C. (2019, September). Perfectionism in Games-Analyzing Playing Behaviors in an Educational Game. In 2019 11th International Conference on Virtual Worlds and Games for Serious Applications (VS-Games) (pp. 1-4). IEEE. (1.5)

[11] Sweetser, P., \& Wyeth, P. (2005). GameFlow: a model for evaluating player enjoyment in games. Computers in Entertainment (CIE), 3(3), 3-3. (3.3)3

[12] Griffiths, Mark. (2010). Does video game addiction really exist?. Education Today: Quarterly Journal of the College of Teachers. 60. 28-32.
[13] Wang, H., \& Sun, C. T. (2011, September). Game reward systems: Gaming experiences and social meanings. In DiGRA conference (Vol. 114)

[14] Flunger, R., Mladenow, A., \& Strauss, C. (2017, December). The free-to-play business model. In Proceedings of the 19th International Conference on Information Integration and Web-based Applications \& Services (pp. 373-379). (1.12)

[15] Artificial Scarcity, Wikipedia, Retrieved From https://en.wikipedia.org/wiki/Artificial_scarcity

[16] Hamari, Juho, and Vili Lehdonvirta. "Game design as marketing: How game mechanics create demand for virtual goods." International Journal of Business Science \& Applied Management 5.1 (2010): 14-29.

[17] Sun, E., Jones, B., Traca, S., \& Bos, M. W. (2015, April). Leaderboard position psychology: counterfactual thinking. In Proceedings of the 33rd Annual ACM Conference Extended Abstracts on Human Factors in Computing Systems (pp. 1217-1222).

[18] Hodent, C. The gamer's brain.

[19] Lin, L., Parmar, D., Babu, S. V., Leonard, A. E., Daily, S. B., \& Jörg, S. (2017, September). How character customization affects learning in computational thinking. In Proceedings of the ACM Symposium on Applied Perception (pp. 1-8)

[20] Turkay, S., \& Kinzer, C. K. (2014). The effects of avatar-based customization on player identification. International Journal of Gaming and ComputerMediated Simulations (IJGCMS), 6(1), 1-25.

[21] De Lope, R. P., Medina-Medina, N., Soldado, R. M., García, A. M., \& Gutiérrez-Vela, F. L. (2017, September). Designing educational games: Key elements and methodological approach. In 2017 9th International Conference on Virtual Worlds and Games for Serious Applications (VS-Games) (pp. 63-70). IEEE.

[22] Longstreet, C. S., \& Cooper, K. (2014, July) Curriculum design factors for constructing serious educational game: A learning objective centric approach. In 2014 Computer Games: AI, Animation, Mobile, Multimedia, Educational and Serious Games (CGAMES) (pp. 1-5). IEEE.

[23] Papadimitriou, S., Chrysafiadi, K., \& Virvou, M. (2019, August). Evaluating the use of fuzzy logic in an educational game for offering adaptation. In 2019 International Conference on Computer, Information and Telecommunication Systems (CITS) (pp. 1-5). IEEE.

[24] De Lope, R. P., \& Medina-Medina, N. (2016, September). Using UML to Model Educational Games. In 2016 8th International Conference on Games and Virtual Worlds for Serious Applications (VS-GAMES) (pp. 1-4). IEEE. 